\title{
BIOMARCADORES COMO HERRAMIENTAS PARA LA DETERMINACIÓN DEL ORIGEN DE EMANACIONES DE HIDROCARBUROS: SURGENCIA DE LA LIBERTAD, ECUADOR, AGOSTO 2016
}

\author{
BIOMARCADORES COMO FERRAMENTAS PARA DETERMINAR A ORIGEM DAS \\ EMANAÇÕES DE HIDROCARBONETOS: VAZAMENTO DE ÓLEO EM LA LIBERTAD, \\ ECUADOR, AGOSTO DE 2016
}

Erika LORENZO GARCÍA, Manuel MARTÍNEZ SANTANA, Antonio MORATO MEDINA
Universidad Estatal Península de Santa Elena. Avenida Principal La Libertad - Santa Elena, La Libertad, Equador. E-mails: elorenzo@upse.edu.ec; mmartinez@upse.edu.ec; antonio.morato@upse.edu.ec

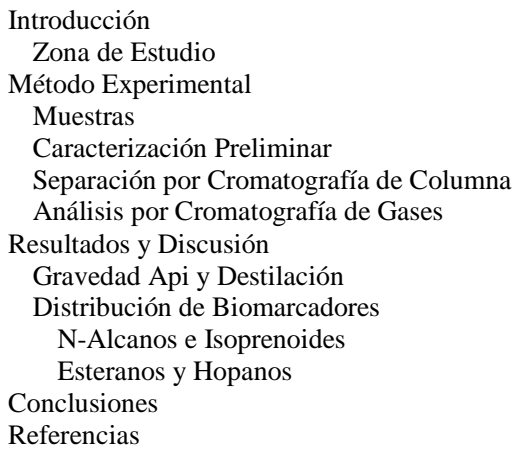

RESUMEN - La Península de Santa Elena (Ecuador) es conocida desde tiempos coloniales por la presencia de menes. A finales de agosto de 2016, una surgencia apareció en una playa del Malecón de La Libertad, cercana a una estación de combustibles. Las relaciones de campo, así como las características fisicoquímicas de la emanación no permitieron esclarecer su probable naturaleza antrópica. El propósito de este trabajo es establecer el origen de la emanación a través del estudio de la distribución de biomarcadores, para lo cual fueron tomadas muestras representativas, fraccionadas por cromatografía de columna, y analizada la distribución de alcanos normales, isoprenoides, esteranos y hopanos. La distribución de isoprenoides y la relación pristano/fitano señalan una correlación genética con los crudos de campos cercanos. Por otra parte, la presencia de esteranos y terpanos tri-, tetra- y pentacíclicos, son incoherentes con un origen antrópico, ya que su alto peso molecular impediría hallarlos en las fracciones ligeras de un producto refinado de origen antrópico.

Palabras clave: Mene, Antrópico, Emanación, Ecuador, Contaminación en playa.

\begin{abstract}
The Peninsula of Santa Elena (Ecuador) is known since colonial times for the presence of seepage oils. At the end of August 2016, a seepage oil appeared on a beach on the La Libertad Coastal Boulevard, near a fuel station. The field relationships, as well as the physicochemical characteristics of the oil emanation did not allow clarifying its probable anthropic nature. The aim of this work is to establish the origin of the seepage oil through the study of the distribution of biomarkers, for which representative samples were taken, fractionated by column chromatography, and analyzed the biomarkers distribution (n- and iso-alkanes, steranes and hopanes). The distribution of isoprenoids and the pristane / phytane relationship are pointing towards a genetic correlation with crude oils from nearby Ancon oilfield. On the other hand, the presence of tri-, tetra- and pentacyclic steranes and terpanes are incoherent with an anthropic origin, since their high molecular weight would prevent them from being found in the light fractions of a refined product of anthropic origin.
\end{abstract}

Keywords: Oil seepage, Anthropic, Emanation, Ecuador, Beach contamination.

\section{INTRODUCCIÓN}

Los biomarcadores son compuestos orgánicos fósiles presentes en muestras geológicas sedimentarias ricas en materia orgánica. Estos productos conservan la estructura básica de sus moléculas precursoras de origen biogénico, a través del registro geológico, por lo que permiten correlacionar crudos entre sí, y crudos con las rocas generadoras (Peters et al., 2005). Estas correlaciones se basan en aspectos genéticos, lo que a su vez permite relacionar los caminos de migración del petróleo con los depósitos de los mismos.

Por este motivo, los biomarcadores constituyen una herramienta valiosa en la exploración petrolera. Cuando se cuenta con un cierto número de manifestaciones de hidrocarburos, se puede definir la presencia de familias de crudos, así como lograr establecer la existencia de diferentes rocas madre y/o variaciones de madurez en el petróleo. Por otra 
parte, el conocimiento de la distribución de los biomarcadores en muestras geológicas permite inferir el medio ambiente de depósito de la materia orgánica, así como la madurez alcanzada (Hill et al., 2007).

En esta comunicación se presentan los resultados del estudio de la distribución de biomarcadores en muestras colectadas de una emanación surgida en la playa más oriental del Malecón de la ciudad de La Libertad (Figura 1), en la Península de Santa Elena, Ecuador. La surgencia apareció a finales del mes de agosto de 2016, caracterizándose por su alta volatilidad, fuerte olor a compuestos organosulfurados y muy baja gravedad específica. El objetivo es determinar si la emanación proviene de alguna fuga derivada de una estación de gasolina cercana $(100 \mathrm{~m})$, o si es una nueva emanación natural que se suma a las numerosas ya reportadas en la región.
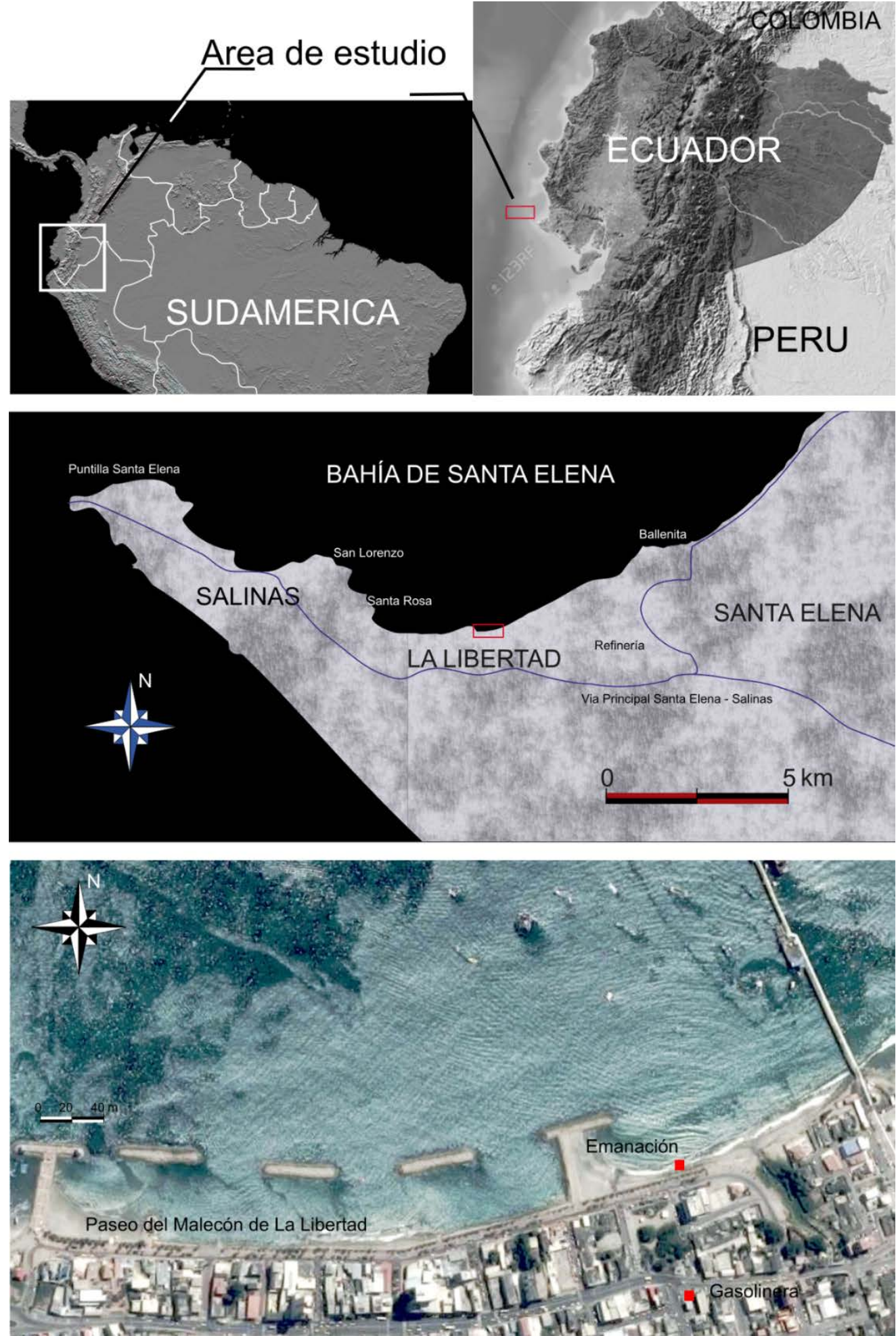

Figura 1. Ubicación del lugar de aparición de la emanación. 


\section{Zona de Estudio}

Toda la región de la Península de Santa Elena y alrededores es conocida desde tiempos coloniales por la presencia de manifestaciones naturales de petróleo; de hecho, compañías inglesas se establecieron en la región a comienzos del siglo $\mathrm{XX}$ dando inicio a la producción petrolera en esta nación.

El Golfo de Guayaquil, junto a la Península de Santa Elena, la región de la desembocadura del río Guayas y la costa del Canal de Jambelí conforman una gran cuenca terciaria, que puede denominarse en forma general como Cuenca del Golfo de Guayaquil (Litton Resources Group, 1986). Por razones históricas, también son denominadas como cuencas a las subdivisiones presentes en ellas: las subcuencas Esperanza, Jambelí, y Progreso; también es incluido el Alto de Santa Elena como un elemento morfotectónico del Golfo (Figura 2).

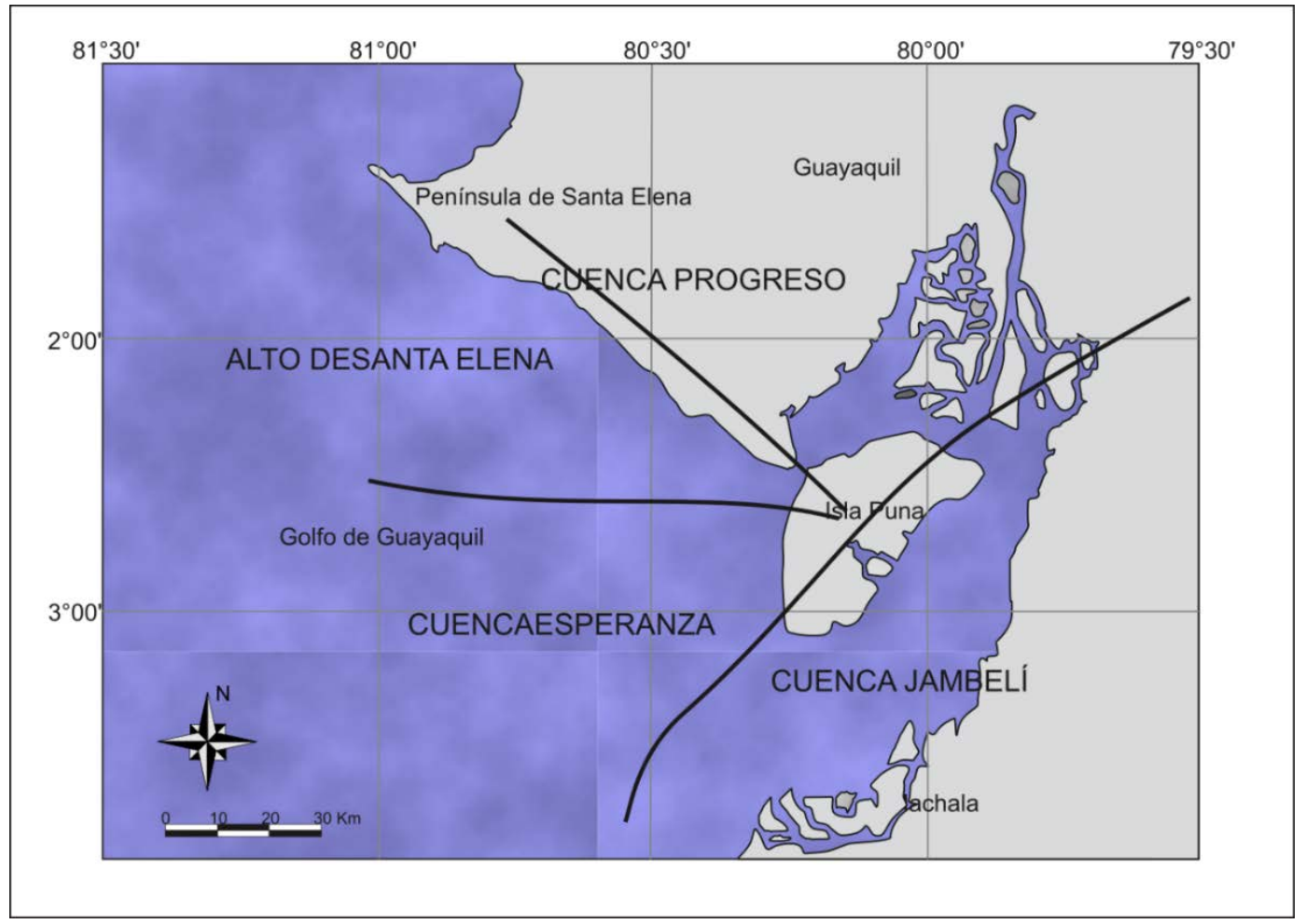

Figura 2. Ubicación de las cuencas Progreso, Esperanza y Jambelí, en la región de la península de Santa Elena y el Golfo de Guayaquil.

La región de la Península de Santa Elena ha sido dividida en varios bloques petroleros productivos, destacando el bloque Gustavo Galindo Velasco, con un área de 70 km2. Este bloque está dividido en tres zonas, norte, central y sur, y algunos de sus campos más importantes son: Ancón, Petrópolis y Santa Paula (Cobos, 2010). Estos campos poseen crudos de importancia económica para Ecuador, y entre ellos destaca el Campo Ancón, cuyos crudos presentan una gravedad API de $35^{\circ}$ en promedio, lo que permite clasificarlos como crudos livianos (Cobos, 2010).

Las rocas almacén de hidrocarburos en el Campo Ancón y aledaños corresponden a secuencias eocenas del Grupo Ancón, particularmente las Formaciones Socorro y Clay Pebble Beds; también existen reservorios paleocenos del Grupo Azúcar o su equivalente, la Formación Atlanta (Zumba, 2005).
En contexto general, la columna sedimentaria de la Cuenca del Golfo de Guayaquil está constituida por la suma de varias unidades tectonoestratigráficas que descansan sobre un basamento oceánico de edad cretácica (Formación Piñón) (Lorenzo et al., 2017). Desde el punto de vista sedimentario, las diferentes secuencias estratigráficas que conforman la columna característica de la subcuenca Progreso responden a variaciones globales periódicas del nivel del mar (pulsos transgresivos). A excepción de la Formación Zapotal que conserva un marcado carácter continental dada su litología conglomeráticas y areniscosa, el resto de formaciones neógenas responden a caracte-rísticas marinas neríticas (lutitas, areniscas finas, y arcillolitas).

Por otro lado, a causa del levantamiento tectónico del bloque de Santa Elena, sobre las rocas del Eoceno se dispone de forma 
discordante erosivo la Formación Tablazo de edad Pleistoceno, estando ausente toda la unidad neógena.

La unidad paleógena (formaciones eocenas y paleocenas de los Grupos Ancón y Azúcar) presenta un claro carácter arenoso y se encuentra a profundidades muy someras en las localidades costeras del Alto de Santa Elena
(Figura 3a). Estas formaciones constituyen los reservorios más importantes de la región y bien, a causa de su ubicación a poca profundidad, por pérdida de roca sello, por migración a la superficie a través de planos de falla o de los estratos de chert presentes en las líneas de playa de la región (Figura 3b), son frecuentes en la zona afloramientos naturales de hidrocarburos.
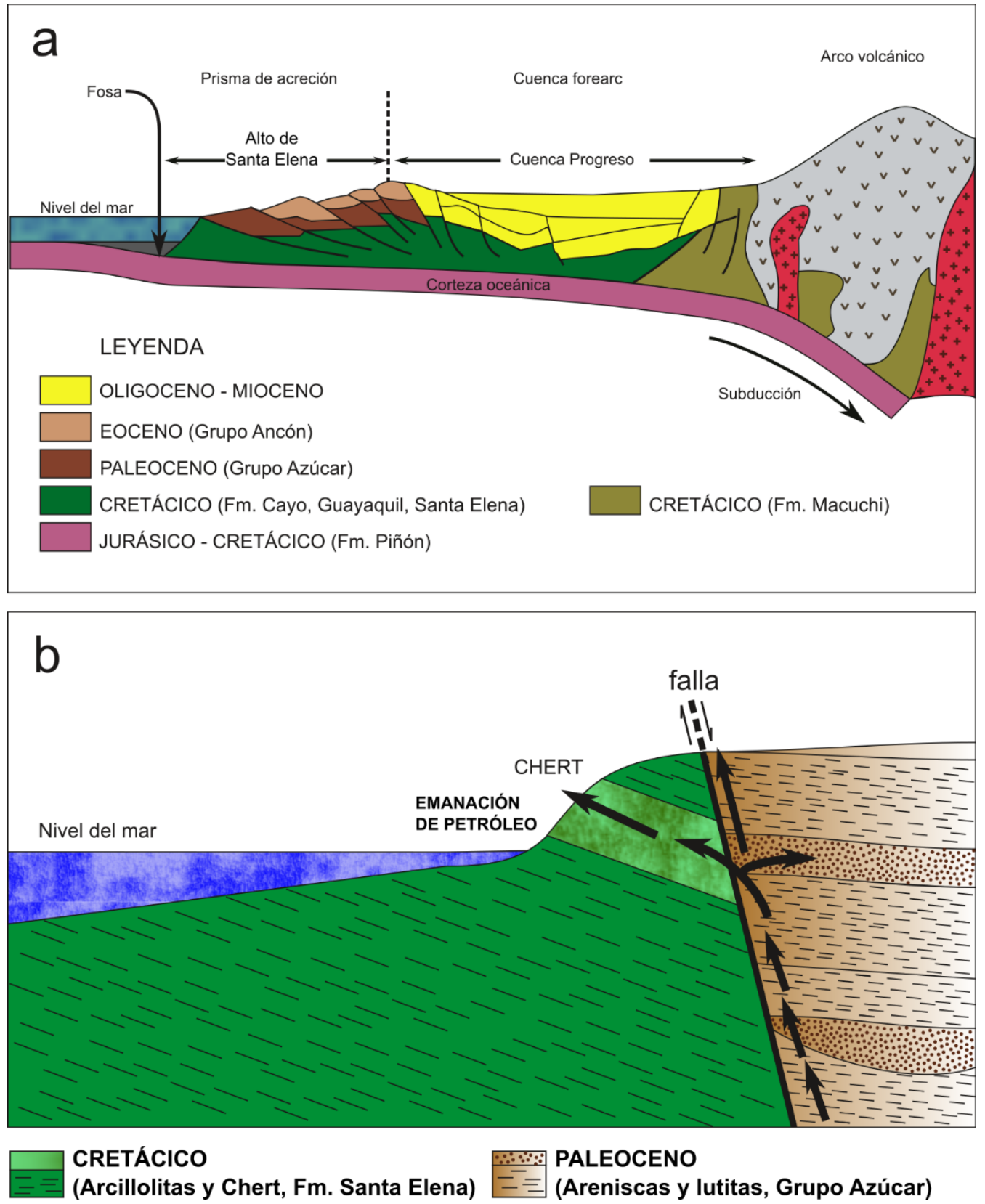

Figura 3. a) Corte geológico que muestra las interrelaciones entre la placa oceánica o Nazca, el arco volcánico, la Cuenca Progreso y el Alto de Santa Elena. b) Ruta propuesta para la migración del crudo en la superficie de la región, aprovechando el contacto Cretácico-Paleógeno.

Varios asentamientos urbanos se han desarrollado y consolidado en el siglo XX en la costa de la Península de Santa Elena, junto a las manifestaciones naturales de hidrocarburos. Debido a la actividad petrolera y las facilidades portuarias, existe en la zona un complejo de refinería y depósito de productos destilados y refinados del petróleo, que con cierta frecuencia experimenta episodios de fuga accidental de hidrocarburos.

A su vez, una avenida muy concurrida de $\mathrm{La}$ Libertad se encuentra a escasos $80-100 \mathrm{~m}$ de la costa norte (Figura 1); en ella se halla una estación de combustible. 
A finales del mes de agosto de 2016, en la playa más oriental adyacente al Paseo del Malecón de La Libertad, apareció una emanación de hidrocarburos en la arena, brotando de la escollera colocada allí como protección del Paseo (2¹3’12” Sur, 8054'27’W, Figura 1). Estima- ciones preliminares de flujo, realizadas en la primera semana de su ocurrencia, arrojaron valores de $20 \mathrm{~mL} / \mathrm{min}$. La emanación de color marrón claro, fluía de forma continua. El flujo mostró variaciones notables a diferentes horas del día (Figura 4).
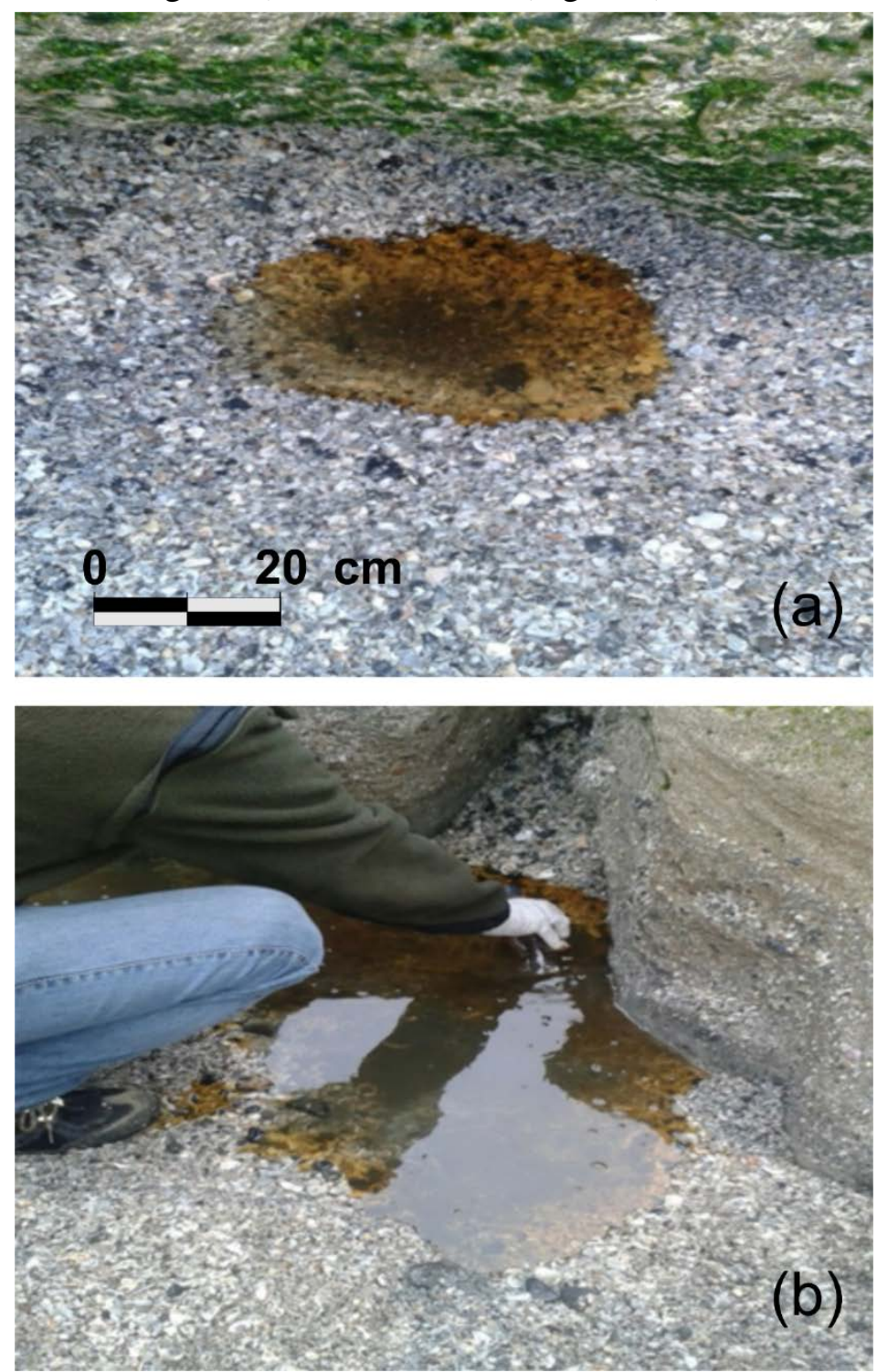

Figura 4. Vista general de la emanación estudiada.

Fue practicada una calicata a la altura del malecón, a aproximadamente $5 \mathrm{~m}$ del brote (Figura 5a). Los sedimentos removidos al fondo de la calicata mostraron indicios de estar impregnados de hidrocarburos. De igual modo, en la plaza central de La Libertad, en proceso de remodelación para el momento de la aparición de la emanación, un pequeño socavón practicado en el margen suroeste evidenciaba indicios de impregnación y olor a hidrocarburos (Figura 5a).

Dos posibles hipótesis se plantearon para establecer el origen de la emanación:

a) Es de origen antrópico (Figura 5b): la presencia de una gasolinera a escasos $100 \mathrm{~m}$ de la surgencia orgánica, el declive natural del terreno, la reducidísima gravedad específica del hidrocarburo ( $51^{\circ}$ API), y el antecedente de un derrame previo colocaban a la estación de combustible como la primera y más evidente causa probable de fuente de la emanación en la playa.

b) Es de origen natural (Figura 5c): la presencia de emanaciones en muchas localidades cercanas, la aparición de una segunda emanación de pequeña expresión a $650 \mathrm{~m}$ al oeste, subacuática, a $20 \mathrm{~m}$ de la playa; y el fortísimo olor a compuestos sulfurados tipo tioles, hacían presumir un posible origen natural. 

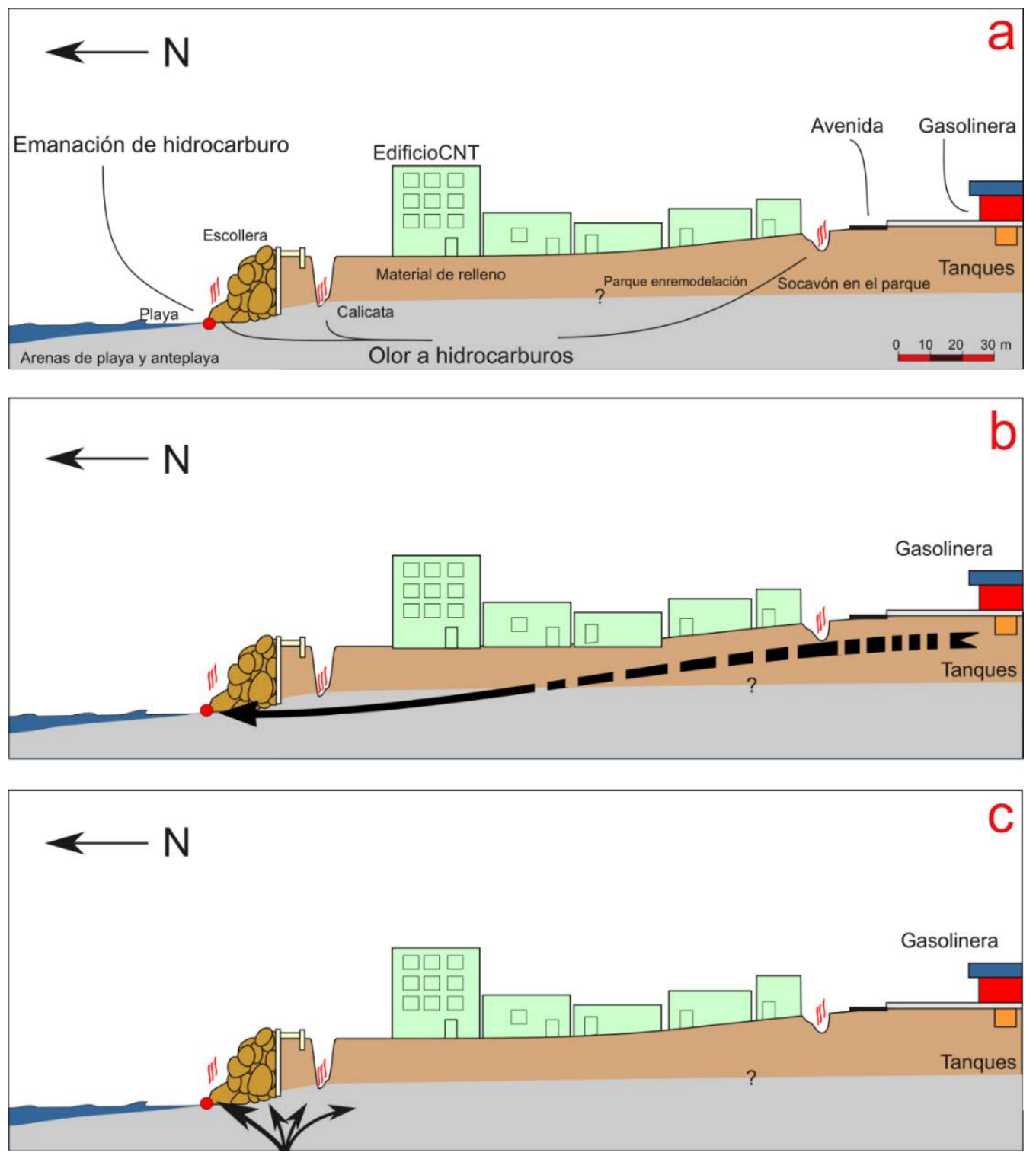

Figura 5. Esquema ilustrativo de la zona, y las hipótesis de trabajo. a) Ubicación de la emanación, el parque cercano y la estación de gasolina. b) Posible origen antrópico para la emanación. b) Origen natural.

\section{MÉTODO EXPERIMENTAL}

\section{Muestras}

Fueron colectadas dos muestras de la emanación, en días diferentes. Además, fue colectada una muestra de sedimentos extraídos en la calicata abierta a escasos metros de la surgencia (Tabla 1).

\section{Caracterización Preliminar}

Una vez en el laboratorio, las distintas muestras fueron preparadas para los ensayos preliminares. En el caso del sedimento, fue necesaria una extracción previa con tolueno.

Las muestras de la emanación (LL-D09 y LLD14) le fueron determinadas sus gravedades API y la curva de destilación ASTM D-86.

\section{Separación por Cromatografía de Columna}

Una porción (50 mg) de cada una de las muestras (emanaciones y el extracto de la calicata) fue separada en las correspondientes fracciones saturadas y aromáticas utilizando una columna de vidrio de $30 \mathrm{~cm}$ de largo y $1 \mathrm{~cm}$ de diámetro. La columna fue empacada con gel de sílice 60 (70230 mesh ASTM) marca Merck, previamente activada a $150{ }^{\circ} \mathrm{C}$ por 10 horas. Los solventes empleados como eluentes fueron n-hexano para obtener los saturados y tolueno para los aromáticos.

\section{Análisis por Cromatografía de Gases}

Las fracciones saturadas fueron analizadas en un cromatógrafo de gases acoplado a espectrómetro de masas (CG-EM) Perkin-Elmer Clarus ${ }^{\circledR}$ SQ 8, equipado con una columna capilar TG-5MS de $60 \mathrm{~m}$ y de longitud y $0,25 \mathrm{~mm}$ de diámetro interno.

Se empleó Helio como gas de arrastre. La temperatura del inyector se mantuvo a $300^{\circ} \mathrm{C}$, utilizándose un volumen de inyección de $1 \mu \mathrm{l}$. La programación de la temperatura fue: temperatura 
inicial, $60^{\circ} \mathrm{C}$ durante $5 \mathrm{~min}$, después se incrementó la temperatura con una razón de $4^{\circ} \mathrm{C} / \mathrm{min}$, hasta llegar a $290^{\circ} \mathrm{C}$; esta temperatura se mantuvo durante 30 minutos.

Se empleó la técnica de Monitoreo de Iones Seleccionados (SIM por sus siglas en inglés) en el detector de masas de ionización electrónica, para obtener los fragmentogramas de relación masa/carga (m/z) 99 (n-alcanos), 191 (terpanos) y 217 (esteranos); también fueron obtenidas las señales totales integradas (“Total Ion Current”, TIC).

Tabla 1. Relación de muestras colectadas.

\begin{tabular}{c|c|c|c}
\hline MUESTRA & FECHA & ORIGEN & OBSERVACIONES \\
\hline LL-D09 & $09 / 09 / 2016$ & Emanación en la escollera & $\begin{array}{c}\text { Marrón oscuro: disperso en agua muestra tonos } \\
\text { levemente rojizos. }\end{array}$ \\
\hline LL-D14 & $14 / 09 / 2016$ & Emanación en la escollera & \\
\hline LL-S14 & $14 / 09 / 2016$ & $\begin{array}{c}\text { Sedimento arenoso colectado al } \\
\text { fondo de la calicata }\end{array}$ & 9 m profundidad. Sedimentos de playa antigua \\
\hline
\end{tabular}

\section{RESULTADOS Y DISCUSIÓN}

\section{Gravedad Api y Destilación}

Los diferentes ensayos practicados sobre las muestras de la emanación colectadas arrojaron los resultados mostrados en la Tabla 2 .

La gravedad API de la emanación corresponde a un condensado. A partir de los datos de la destilación, se construyó la curva de destilación ASTM D-86 (Figura 6a).

La curva de destilación TBP ("True Boiling Point”) obtenida a partir de la conversión correspondiente de la gráfica de destilación ASTM D-86 para las dos muestras (Figura 6b) permite concluir que la emanación está constituida en un 76-80 \% por hidrocarburos del rango de la gasolina, y un 20-24\% a diésel (Fahim et al., 2010). Para propósitos de la variabilidad asociada al muestreo, las diferencias entre ambas muestras no son significativas.

El análisis de la Figura 6b muestra una continuidad clara de las curvas de destilación en ambas muestras.

Para una emanación proveniente de fuente antrópica (por ejemplo, una fuga de los tanques de la gasolinera), se esperaría, por tratarse de un producto refinado, una inflexión clara hacia los $193{ }^{\circ} \mathrm{C}$, punto de corte para discernir entre diésel y gasolina.

Sin embargo, los resultados obtenidos al momento sólo permiten caracterizar a la emanación de forma general. Se requiere una caracterización a nivel molecular que permita establecer en forma inequívoca su origen; y para ello es necesario recurrir al análisis geoquímico molecular.

Tabla 2.- Resultado de los ensayos de laboratorio.

\begin{tabular}{c|c|c}
\hline PARÁMETRO & LL-D09 & LL-D14 \\
\hline Gravedad específica & $0,775 \pm 0,002 \mathrm{~g} / \mathrm{cm}^{3}$ & $0,773 \pm 0,002 \mathrm{~g} / \mathrm{cm}^{3}$ \\
\hline Gravedad API & $51^{\circ} \mathrm{API}$ & $51^{\circ} \mathrm{API}$ \\
\hline Volumen inicial & $100 \mathrm{~mL}$ & $100 \mathrm{~mL}$ \\
\hline Volumen recolectado & $98 \mathrm{~mL}$ & $97 \mathrm{~mL}$ \\
\hline$\%$ Destilado a $70^{\circ} \mathrm{C}$ & $<5 \%$ & $<5 \%$ \\
\hline \% Destilado a $193^{\circ} \mathrm{C}$ & $76 \%$ & $80 \%$ \\
\hline Temperatura al $90 \%$ colectado & $230^{\circ} \mathrm{C}$ & $228^{\circ} \mathrm{C}$ \\
\hline
\end{tabular}



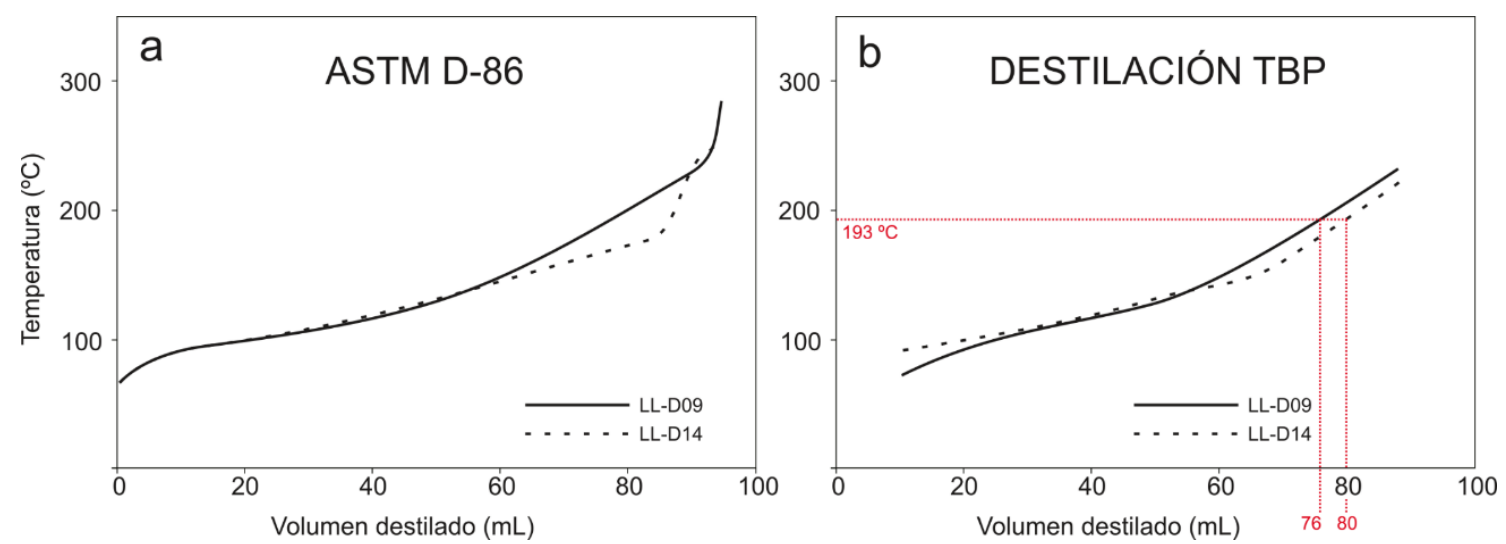

Figura 6: Curvas de destilación para la emanación y el extracto de la calicata. a) Destilación ASTM. b) Destilación TBP.

\section{Distribución de Biomarcadores}

El análisis molecular implica la detección de un conjunto de estructuras orgánicas conocidas inicialmente como fósiles químicos (Eglinton \& Calvin, 1967), luego como marcadores biológicos (Hoffmann et al., 1984) y hoy en día reciben el nombre de biomarcadores (Peters et al., 2005). Los biomarcadores pueden ser: a) hidrocarburos; b) compuestos orgánicos funcionalizados (alcoholes, ésteres, ácidos, cetonas, etc.); c) compuestos heteroatómicos (N-S-O). En el presente trabajo, a fin de llevar a cabo un análisis rápido y efectivo, se trabajó sólo con los biomarcadores del primer tipo, que son fácilmente detectados e identificados por cromatografía de gases acoplada a un detector de masas (GCMS). Los hidrocarburos catalogados como biomarcadores son compuestos orgánicos constituidos exclusivamente por carbono e hidrógeno, cuya estructura composicional y sobre todo su arreglo espacial es producto de un precursor biológico del que proceden. Los más importantes son:

a) Alcanos (tanto lineales, como ramificados y cíclicos)

b) Isoprenoides

c) Esteranos

d) Terpanos

Debido a que la distribución de biomarcadores fue exactamente la misma en ambas muestras de la emanación y en el extracto de sedimento (en esta última muestra sólo cambia la abundancia relativa de los componentes más volátiles, C15-) sólo se mostrarán los resultados de los fragmentogramas correspondientes a la muestra LL-D09.

\section{n-alcanos e Isoprenoides:}

La familia de n-alcanos junto a los isoprenoides correspondientes a la muestra LLD09 pueden ser monitoreados apropiadamente en la relación masa-carga m/z 99 (Figura 7a). También en esta figura aparece el llamado TIC ("Total Ion Current") que integra la contribución de todos los iones generados en el proceso de cromatografía de la muestra (Figura 7b).

El análisis de ambos fragmentogramas permite detectar la presencia dominante de varios isoprenoides, principalmente pristano, fitano, norpristano, y otros isoprenoides de menor peso molecular. La distribución de nalcanos e isoprenoides en la muestra LL-D9 es peculiar con respecto al patrón generalizado presentado por este tipo de compuestos. En el caso que nos ocupa, se muestra una predominancia inusual de los compuestos isoprenoides sobre la de n-alcanos.

Es sabido que la biodegradación, sobre todo en sus etapas iniciales, puede generar una diferenciación entre n-alcanos e isoprenoides (Peters et al., 2005). La casi total ausencia de nalcanos $<$ nC18 puede ser atribuida a alteración bacterial (Gürgey et al. 2007). Sin embargo, en los casos reportados en la literatura no se encuentra un patrón como el obtenido en la surgencia estudiada (Bailey et al., 1973; García et al., 2012; Oblasov et al., 2018). Esta distribución pese a ser particular, es similar a la presentada por crudos analizados procedentes de campos petrolíferos del área cercana SP-47 (Figura 7c). La similitud presentada, apunta a una posible relación genética entre ambos. Por otro lado, la relación entre los isoprenoides Pristano/Fitano tanto en la muestra problema como en el crudo de referencia son virtualmente similares, lo que parece indicar que el origen de la emanación es natural. 


\section{Esteranos y Hopanos:}

Los esteranos conforman un grupo de moléculas que al igual que la familia de los hopanos (triterpanos, terpanos tretracíclicos o pentacíclicos) y otros componentes orgánicos naturales, son propios de crudos. Sin embargo, a causa de sus altos pesos moleculares y puntos de ebullición, no están presentes en las fracciones destiladas del crudo. Por tanto, están ausentes en gasolinas. El ión $217(\mathrm{~m} / \mathrm{z}=217)$ es diagnóstico de este grupo de moléculas (Figura 8a). La Figura 8b muestra el fragmentograma 217 para la emanación, donde se observa esta familia de compuestos, descartando por tanto el origen antrópico propuesto en una de las hipótesis. Igualmente en el fragmentograma 191 se puede observar la presencia de la familia de hopanos, incompatible en cortes de destilación.

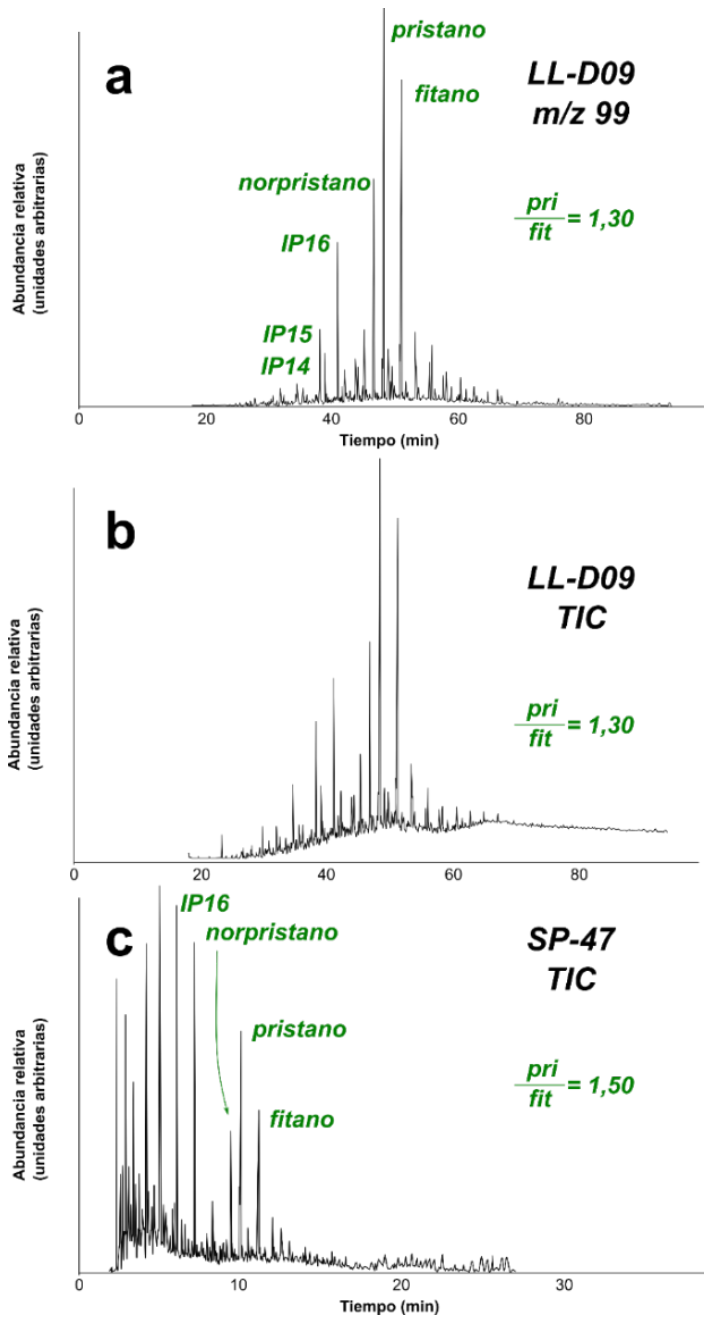

Figura 7. a) Cromatograma de iones totales (“Total Ion Current”, TIC) de la muestra de la emanación. b) Fragmentograma m/z 99. c) Cromatograma TIC del crudo SP-47, del cercano campo Pacoa (Lorenzo et al., 2017).
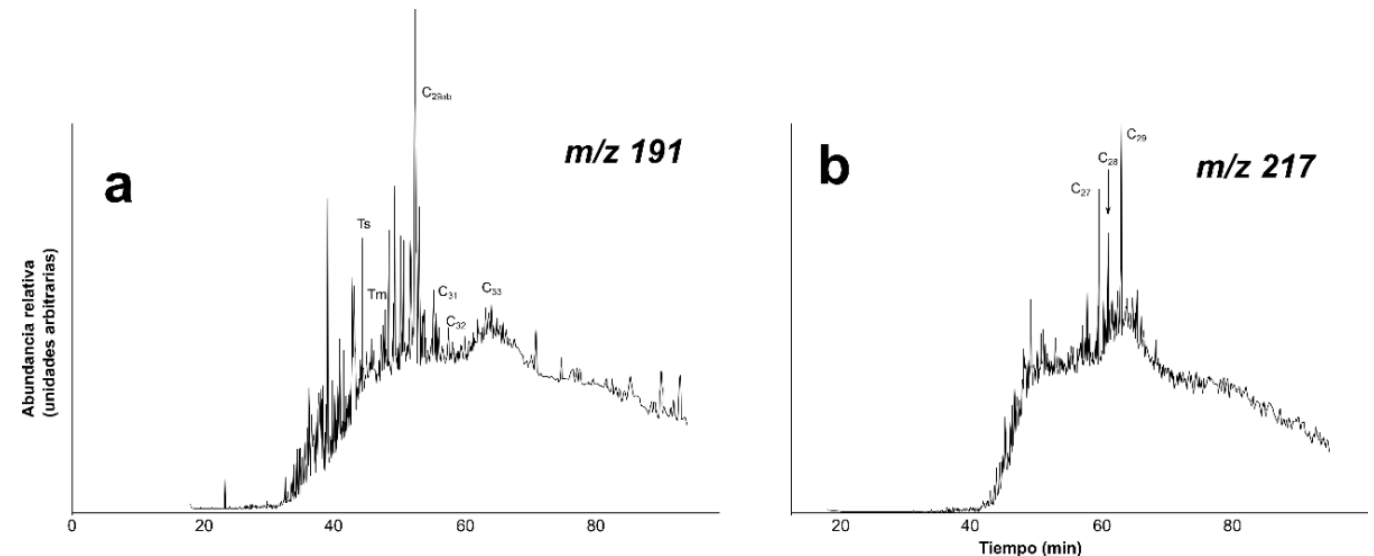

Figura 8: a) Fragmentograma m/z 191, correspondiente a la distribución de hopanos. b) Fragmentograma m/z 217, correspondiente a los esteranos. 


\section{CONCLUSIONES}

Las características preliminares tales como el color, la ausencia de interrupción en la curva de destilación, así como la composición mayoritaria de hidrocarburos en el rango de la gasolina y una pequeña proporción correspondiente a diésel son inconsistentes con la posibilidad de cortes de refinación, lo que permite proponer en primera instancia que la emanación tiene origen natural.

La distribución de n-alcanos e isoprenoides es inusual, sin embargo la similitud con otros crudos producidos en el Campo Santa Paula de la Península de Santa Elena evidencia una clara relación genética entre ambos, confirmando el origen natural de la muestra. La presencia de compuestos de alto peso molecular tales como las familias de los esteranos $u$ hopanos descartan toda posibilidad de que la muestra tuviera origen antrópico. Finalmente, de las dos hipótesis inicialmente propuestas queda descartada la filtración de la gasolinera como fuente de la emanación al pie del Malecón de la Libertad, siendo un afloramiento natural de hidrocarburos de los tantos otros existente en la península dado el subsuelo petrolero de la misma.

\section{REFERENCIAS}

BAILEY, N.J.; JOBSON, A.M.; ROGERS, M.A. Bacterial degradation of crude oil: Comparison of field and experimental data. Chemical Geology, v. 11, p. 203-21, 1973.

COBOS, L. Estudio Integrado Del Golfo de Guayaquil Del Mioceno Al Reciente. Tesis de Grado, Facultad de Ingeniería en Ciencias de la Tierra, Escuela Superior Politécnica del Litoral. Facultad de Ingeniería en Ciencias de la Tierra, 2010.

EGLINTON, G. \& CALVIN, M. Chemical Fossils. Scientific American, v.216, p. 32-43, 1967.

FAHIM, M.A.; AL-SAHHAF, T.; ELKILANI, A. Fundamentals of Petroleum Refining. Amsterdam: Elsevier, 516 p., 2010.

GARCÍA, G.; INFANTE, C.; LÓPEZ, L. Biodegradación de Un Crudo Mediano en suelos de diferente textura con y sin agente estructurante. Bioagro, v. 24, n. 2, p. 93-102, 2012.

GÜRGEY, K.; SIMONEIT, B.; BATI, Z.; KARAMANDERESI, H.; VAROL, B. Origin of Petroliferous Bitumen from the Büyük Menderes-Gediz Geothermal Graben System, Denizli - Sarayköy, Western Turkey. Applied Geochemistry, v. 22, n.7, p. 1393-1415, 2007.

HILL, R.; JARVIE, D.; ZUMBERGE, J.; HENRY, M.; POLLASTRO, R. Oil and gas geochemistry and petroleum systems of the Fort Worth Basin. AAPG Bulletin, v. 91, n. 4, p. $445-473,2007$.

HOFFMANN, C.; MACKENZIE, A.; LEWIS, C. A Biological Marker Study of Coals, Shales, and Oils from the Mahakam Delta, Kalimantan, Indonesia. Chemical Geology, v. 42, p. 123, 1984.
LITTON RESOURCES GROUP. The Gulf o Guayaquil Report Study, 1986.

LORENZO, E.; ROCA, W.; MARTÍNEZ, M.; MORATO, A.; ESCANDÓN, P.; ÁLVAREZ, C. Correlación Geoquímica entre crudos y rocas del Sistema Petrolero de la Península de Santa Elena y el Golfo de Guayaquil. Boletín de Geología, v. 40, n. 1, p. 31-42, 2017.

OBLASOV, N.V.; GONCHAROV, I.V.; SAMOILENKO, V.V.; GRASS, G.W. VAN. Biodegradation in the Nkh 3-4 Reservoir at Vankor Field (West Siberia Basin, Russia) Is Strongly Controlled by Rock Properties. Organic Geochemistry, v. 119, p. 36-49, 2018.

PETERS, K.; WALTERS, C.; MOLDOWAN, M. The Biomarker Guide, Vol. I. 2nd. Cambridge, Cambridge University Press, 702 p., 2005.

ZUMBA, N. Interpretación Geológica-Sísmica 2D en el Área San Vicente-Morillo del Bloque ESPOL. Tesis de Grado, Facultad de Ingeniería en Ciencias de la Tierra, Escuela Politécnica Superior del Litoral, 185 p., 2005 